\title{
Comparative study of deterioration of composite due to ice formation using strain, electromechanical impedance and guided
} waves

\author{
Rohan Soman ${ }^{1,}{ }^{*}$, Pawel Malinowski ${ }^{1}$, and Wieslaw Ostachowicz ${ }^{1}$ \\ ${ }^{1}$ Institute of Fluid Flow Machinery, Polish Academy of Sciences, Fiszera 14, Gdansk. 80-231, Poland
}

\begin{abstract}
Glass fibre reinforced plastic (GFRP) composites are finding increasing application in aerospace structures. The monitoring of these structures is not only necessary but also mandatory by the safety codes. The present state of the art allows isolation of damage and the quantification of damage is the next challenge. The quantification may allow for better maintenance scheduling and as a result lower downtime for airplanes, which makes it significant in the aerospace discipline. It is known that the moisture absorption in the composites affects its mechanical properties. The moisture can interact with the composite sample in the gaseous, liquid or solid phase. The mechanism for deterioration of the composite due to the moisture depends on the phase in which the interaction occurs. The paper thus investigates the interaction of water absorbed by the sample in the solid phase. The GFRP sample is exposed to low temperatures after the ingress of the moisture. The sample is studied using three distinct damage detection methods namely, Electromechanical Impedance technique (EMI), Guided waves method (GW) and NA based technique based on measured strains. The aim of the research is to compare the performance of the three methods for the assessment of the deterioration of the composite samples due to ice. The EMI, GW and strain based method have been shown to be sensitive to moisture induced deterioration. In addition to the detection of deterioration, the use of measured strains provides an intuitive way for the quantification of the moisture induced deterioration in the sample.
\end{abstract}

\section{Introduction}

Glass fibre reinforced plastics (GFRP) offer have high strength and stiffness to weight ratio, low cost, and give an opportunity to tailor their properties to our requirements. Due to these advantages, they are finding applications in different industrial sectors from aerospace, renewable energy, marine, etc. The expansion of the range of applications is accompanied by demand for larger, and more complex composite components (such as wind turbine blade, aircraft parts). This increase in the complexity of components leads to

\footnotetext{
*Corresponding author: rsoman@imp.gda.p1
} 
difficulties in manufacturing and maintenance. Also the applications in wind and aerospace industry expose the material to challenging ambient conditions. The structures are often exposed to freezing conditions, and ice formation is a common occurrence in both the sectors. The anomalous behaviour of water has been a blessing for all life forms and may be one of the key reasons that there is life on the earth. But for the structures it might be a curse. The volume of water reduces as the temperature decreases until $4^{\circ} \mathrm{C}$. But then increases until the formation of ice. Thus, water in the liquid phase has a lower viscosity and can enter the voids inside the composites. But in icing conditions, the water expands and, due this expansion, the structure experiences internal stresses which might lead to damage of the structure. This increase in the internal stresses often leads to cracks, and may also result in the misalignment of members which affect the global strength of the composite by up to $40 \%$. [1]. Thus, it is necessary to study the damage mechanism, and the detection of deterioration caused due to the ice formation using the SHM techniques.

Most of the studies found in literature employ some destructive tests for studying the effects of temperature on the mechanical properties and quantifying them, but the number of studies is limited to only one type of assessment per sample due to the destructive nature of the tests. Different tests on different samples may not be used for the assessment of these methods due to the high degree of variability in the sample preparation that is encountered [2]. In order to overcome this variability the present work makes use of 3 non-destructive evaluation techniques (electromechanical impedance (EMI), guided waves (GW) and strain measurement based methods), which allow the comparative assessment of the methods on the same sample. The effect of icing in two distinct cases is studied. For the first case, the GFRP sample is immersed in water till saturation is achieved and then the sample is exposed to sub-zero temperatures for ice formation to occur. In the second case the sample with a slot is prepared. Water is filled in the slot, and the effect of the phase change of the water on the GW and EMI is studied.

\section{Theoretical Background}

Three different techniques were chosen based on their sensitivity to detection of deterioration due to moisture. The overview of the three techniques is explained here.

\subsection{Electromechanical Impedance (EMI)}

Some materials like lead zirconate titanate (PZT) show electromechanical coupling. The property can be used for producing actuators and sensors. The PZT transducers are glued to the specimen and powered with alternating voltage. These transducers change their frequency characteristics of electrical impedance with the changes in the mechanical properties of the specimen. Any change in the material will cause local change of stiffness, mass and damping properties which in turn modifies the impedance plots. The EMI method is sensitive to ambient moisture absorption and has been used for the detection of moisture deterioration of composites. The phenomenon can be expressed in equations (1) and (2) [3]:

$$
\begin{aligned}
& D_{i}=\varepsilon_{i j}{ }^{T} E_{j}+d_{i m} T_{m} \\
& S_{k}=d_{j k} E_{j}+s_{k m}{ }^{E} T_{\mathrm{m}}
\end{aligned}
$$

where, $D$ is the electric displacement, $S$ the mechanical strain, $E$ the electric field and $T$ the mechanical stress. $\varepsilon$ is the complex electric permittivity of the PZT material at constant stress, $d$ is the piezoelectric strain coefficients (or constants) and $s$ the complex elastic compliance at constant electric field. The superscripts $T$ and $E$ indicate that the quantity has 
been measured at constant stress and constant electric field respectively. In $d_{i m}$ and $d_{j k}$, the first subscript denotes the direction of the electric field and second, the direction of the associated mechanical strain.

\subsection{Guided waves based method (GW)}

The composite specimens under investigation are plate like structures, and as such the guided waves in plates will exist in the form of Lamb waves. Lamb waves can travel large distance and hence may be used for the monitoring of the specimen. The guided waves travel in the specimen and are reflected from boundaries and discontinuities in the system. Lamb waves can exist in two forms, the symmetric modes and antisymmetric modes. For a given frequency thickness product, there can exist a number of modes of each type. The processing of these samples is very difficult, and thus, Lamb waves are suitable for thin plates. The symmetric modes are designated S0, S1, S2 etc. and resemble the axial waves while the antisymmetric modes are designated A0, A1, A2 etc. and resemble the flexural modes.

The equations for the Lamb waves for symmetric and antisymmetric mode are given by equation 3 and equation 4 [4].

$$
\begin{aligned}
\tan (p d) / \tan (q d) & =-\left\{\left(\xi^{2}-q^{2}\right)^{2} / 4 \xi^{2} p q\right\} \\
\tan (p d) / \tan (q d) & =\left\{4 \xi^{2} p q /\left(\xi^{2}-q^{2}\right)^{2}\right\}
\end{aligned}
$$

\subsection{Neutral Axis based Method}

The applied load results in bending strains in the beam. As seen in Fig. 1, the bending strain is tensile $\left(\varepsilon_{t}\right)$ at one of the surfaces, and compressive $\left(\varepsilon_{\mathrm{b}}\right)$ at the opposite surface. In between, this top and bottom edge is the NA of the cross section. The NA of the section is a function of the flexural rigidity of the structure, and does not depend on the applied bending loads, thus by, measuring the strains at the opposite edges of the beam, the NA can be located, using equation 5 [5].

$$
N A=\varepsilon_{t} /\left(\varepsilon_{t}-\varepsilon_{b}\right)=y_{n} / h
$$

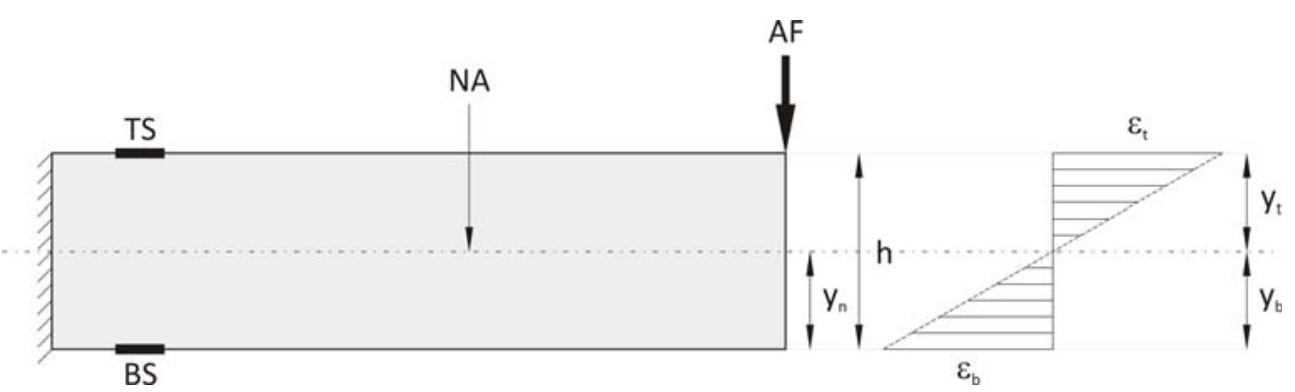

Fig. 1. Flexural strain distribution over beam cross-section [6].

The NA location in turn can be used as a damage sensitive feature. If the change in the NA position is greater than a certain threshold, one can conclude that the structure is damaged. The Fig. 2 explains the abbreviations used and the concept. The NA location can thus be estimated based on the strain measurements. The authors in their previous work have shown that through the use of Kalman Filter (KF) NA can be tracked accurately in the presence of measurement noise, temperature variation and changing loads $[6,7]$. 


\section{Experimental Setup}

As mentioned before, 2 distinct cases were studied. Two different specimens were used for the two studies as shown in Fig. 2.

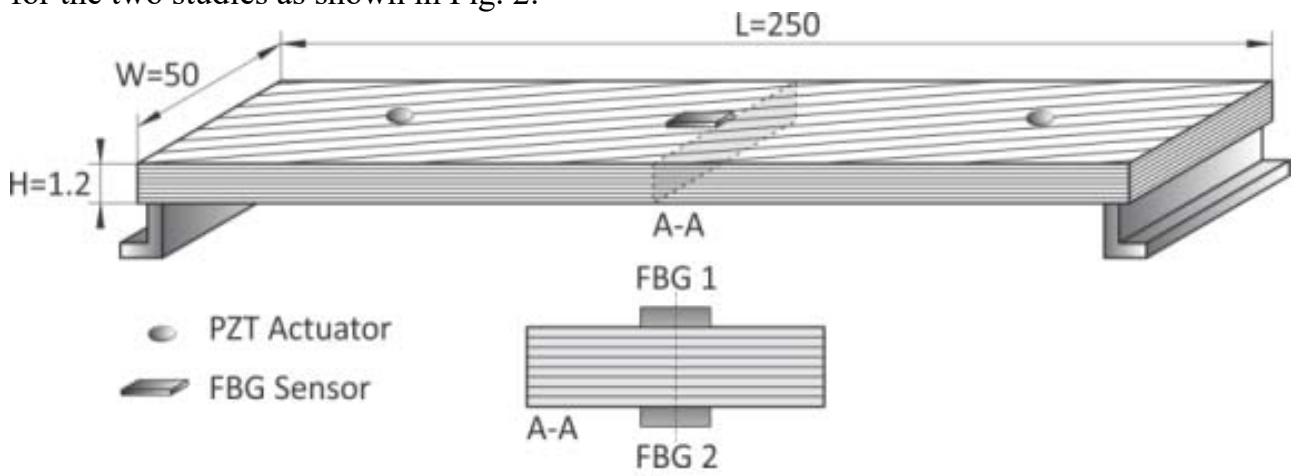

(a)

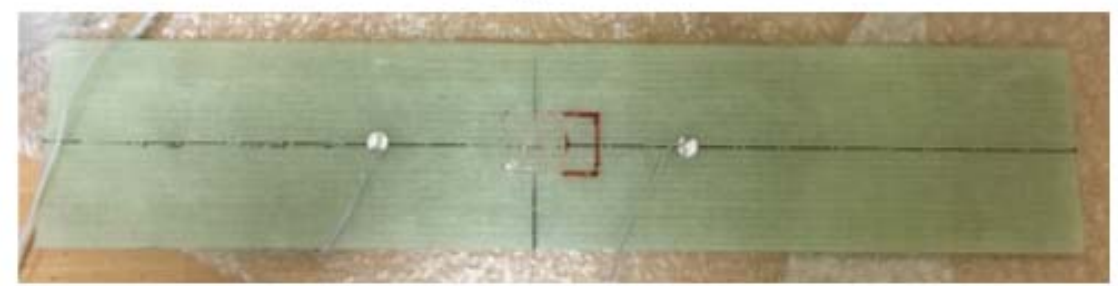

(b)

Fig. 2. Beam samples used for the study a) sample 1 b) sample 2

The Figure 2 (a) shows the dimensions of one sample. The details of the experiments conducted are reported in [8]. The only difference in the experiment, was the conditions the beam was exposed to. The box with water and the samples was placed in the ambient conditions (sub-zero), winter in Poland for 2 weeks. The temperature conditions were not recorded due to the failure of the weather station. But at the end of the 2 weeks period, the entire sample was covered with ice. The measurements will all the three conditions were then conducted and compared with the baseline. The figure 2 (b) shows the sample used to study the effect of phase change on the EMI and GW techniques. The plate has dimensions $500 \times 100 \mathrm{~mm}$ with a $30 \mathrm{~mm}$ square slot $25 \mathrm{~mm}$ off center. The square slot has a depth of half the plate thickness. Two PZT's are placed such that the edges of the slot are $60 \mathrm{~mm}$ from each PZT. The influence of ice and water was studied by comparing the measurements to the baseline. $3 \mathrm{ml}$ of water was added to the slot and the measurements were taken. The plate was then placed in the refrigerator to study the effect of ice. The measurements were repeated with ice.

\section{Results and Discussion}

\subsection{Sample 1:}

The samples immersed in water were investigated with the three methods. The Fig. 3 shows the change in GW propagation for the dry condition, saturated with water condition and after the sample was frozen. Two observations can be made, there is a delay between excitation and wave arrival in case of water and ice. This delay although expected, cannot 
be measured with certainty due to the limited sensitivity of the apparatus. The other observation is the attenuation. This attenuation is significant and for the time period shown in Fig. 3 is around $8 \%$ for the water and $12 \%$ for the ice. This result too is in agreement with the results reported in the previous work [9].

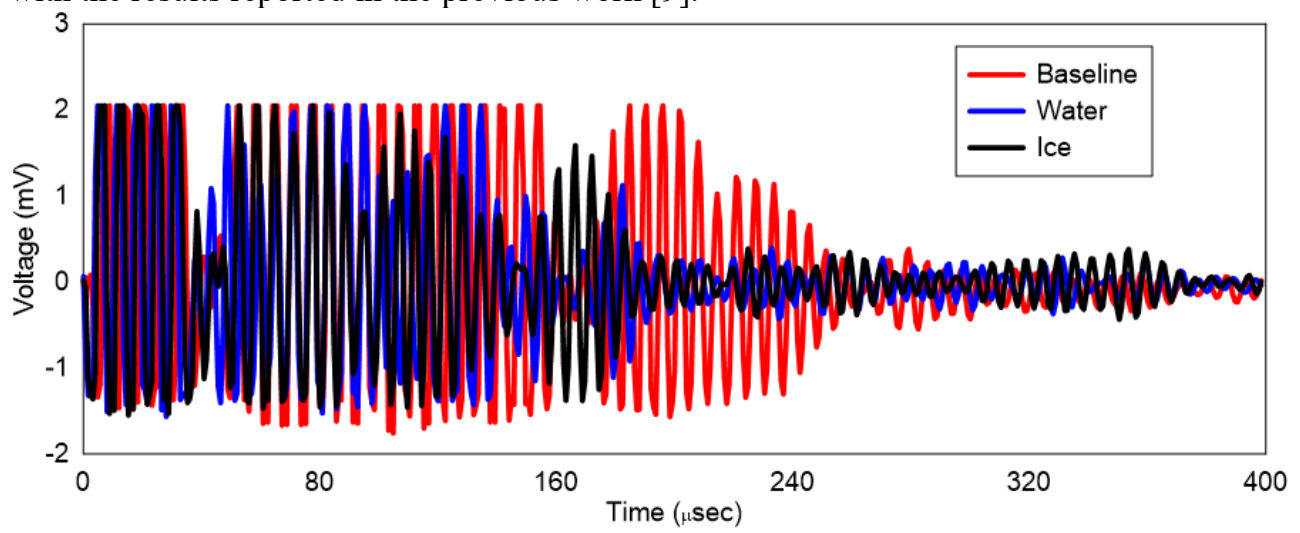

Fig. 3. GW propagation for dry sample, saturated and after icing.

The EMI technique results are presented in Fig. 4 and Fig. 5. The low frequency changes are observed better in the resistance plot, while the conductance plots shows changes at high frequencies.

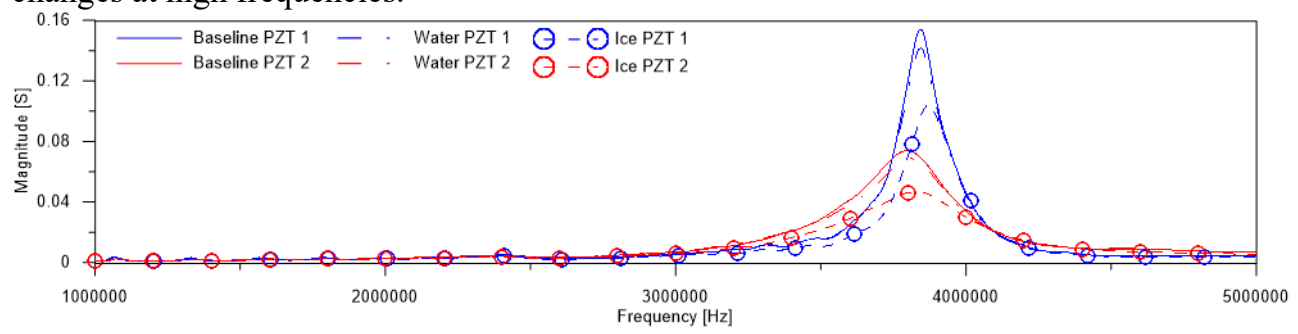

Fig. 4. Conductance plot for higher frequencies for EMI technique

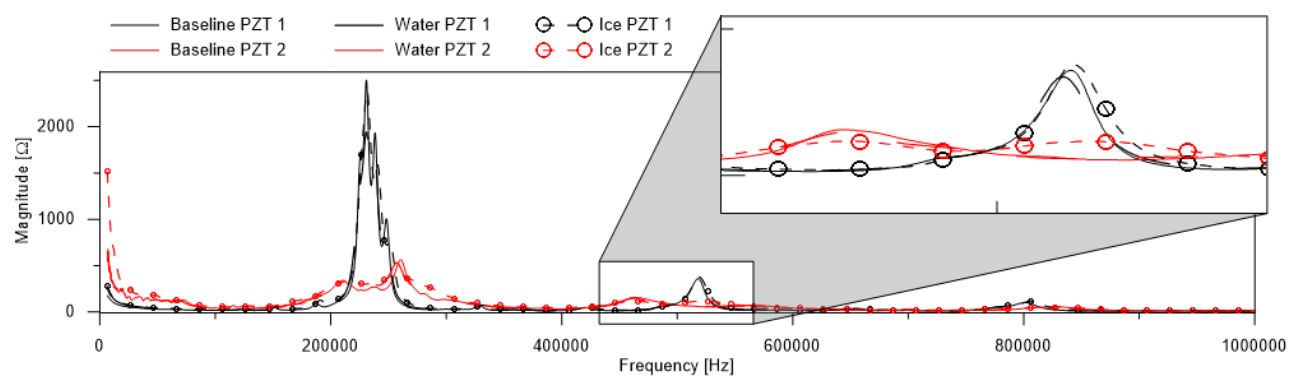

Fig. 5. Resistance plot for lower frequencies for EMI technique

As can be seen in the conductance plot, for the dry plate and the saturated plate, there is no appreciable difference for the EMI technique. There is a slight reduction in the amplitude of vibration which can be attributed to additional damping. On the other hand for the icing case there is a significant change in the amplitude as well as the frequency. This frequency increase is unexpected, as the added weight of water is expected to move the peaks towards the left. One possible reason for the rightward shift may be due to the change in temperature. Similar trend is seen for the resistance plots at the lower frequencies. 
For the strain based study, the results of the NA tracking are reported in Table 1. As can be seen the threshold of $1 \%$ is exceeded in all conditions [5]. The results although need to be treated with due care. The NA based technique has been shown to be insensitive to applied loads and should yield stable NA estimates under different loads [5-8]. As the NA estimate for the different loading conditions is not stable, this points to systematic errors in the strain measurements. As a result the health assessment of the beam cannot be made using the strain based technique. This phenomena is similar to the one reported in [8] by the authors, where the glue turned brittle due to the interaction with water and the temperature, and as such the strain measurements were not reliable. Although the results obtained are not satisfactory, the study supports the conviction of the authors that one single technique may not be suitable for the complete SHM of the system. A combination of two or more techniques may be necessary for the damage assessment of the structure.

Table 1. NA location under two loading cases for scenarios

\begin{tabular}{|c|c|c|c|c|}
\hline Scenario & \multicolumn{4}{|c|}{$\begin{array}{c}\text { NAE location } \\
\text { (\% NAE) }\end{array}$} \\
\hline Orientation & \multicolumn{2}{|c|}{ PZT -up } & \multicolumn{2}{c|}{ PZT -down } \\
\hline & $100 \mathrm{~g}$ & $200 \mathrm{~g}$ & $100 \mathrm{~g}$ & $200 \mathrm{~g}$ \\
\hline Baseline & 0.401 & 0.401 & 0.401 & 0.401 \\
& $(0)$ & $(0)$ & $(0)$ & $(0)$ \\
\hline Water & 0.397 & 0.406 & 0.424 & 0.398 \\
& $(1.00)$ & $(1.25)$ & $(5.74)$ & $(0.75)$ \\
\hline Ice & 0.382 & 0.393 & 0.468 & 0.434 \\
& $(4.73)$ & $(1.99)$ & $(16.66)$ & $(8.22)$ \\
\hline
\end{tabular}

\subsection{Sample 2:}

In addition to the comparative assessment using the three methods. Studies were also undertaken to study the effect of phase change, and how the phase change is reflected in the EMI and GW techniques. Due to the uncertainty with the glue performance, the strain measurements were not performed for the sample 2. The sample dimensions have been given in section 2. The Fig. 6 and Fig. 7 show the low frequency and high frequency plots for the plate under the water and icing conditions.

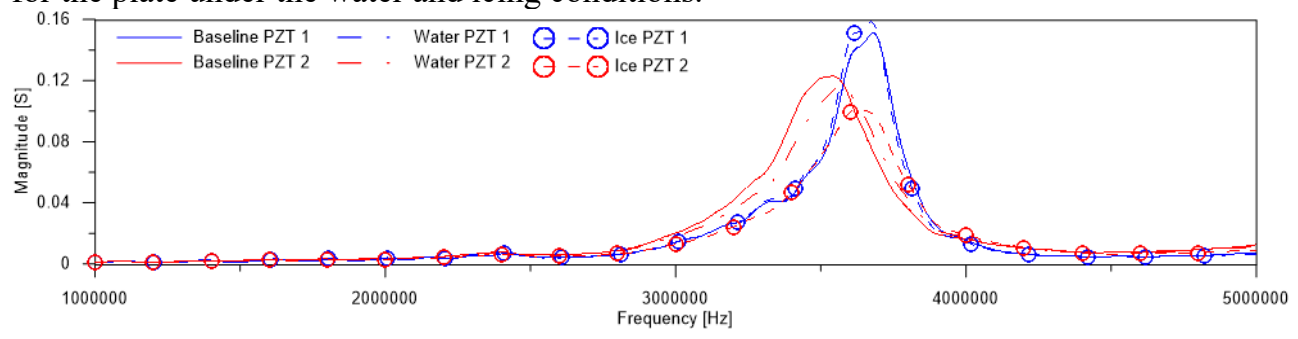

Fig. 6. Conductance plot for higher frequencies for EMI technique for slotted plate

For the slotted plate there is no appreciable difference between the baseline and plate with water. The quantity of the water added was negligible $(3 \mathrm{ml})$ and hence does not affect the frequencies significantly. The icing conditions although do bring a change in the properties. The change may be due to the change in the temperature or the different behaviour of the plate due to ice. The temperature compensation studies have been identified as work for the future. For the quantification of the change in the three conditions, the Root mean square (RMS) metric [10] was used for the resistance measurements in the range of $0.5 \mathrm{MHz}$ to $1 \mathrm{MHz}$ as shown in Fig 8. 


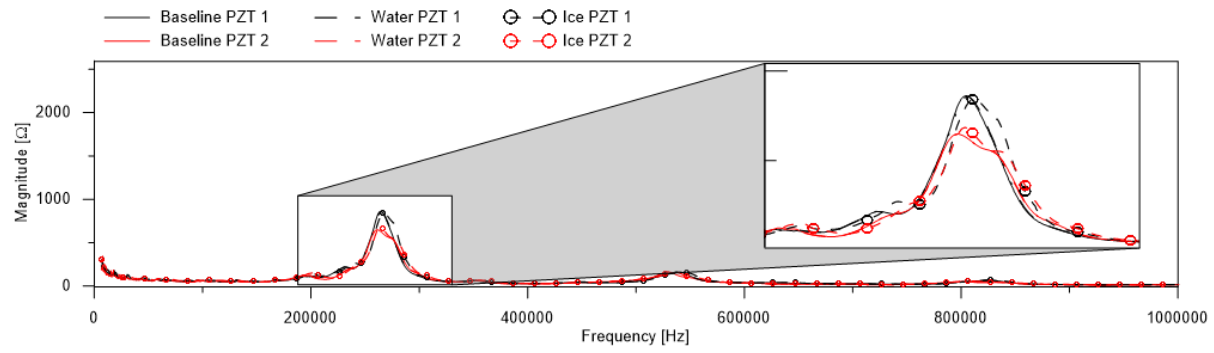

Fig. 7. Resistance plot for lower frequencies for EMI technique for slotted plate.

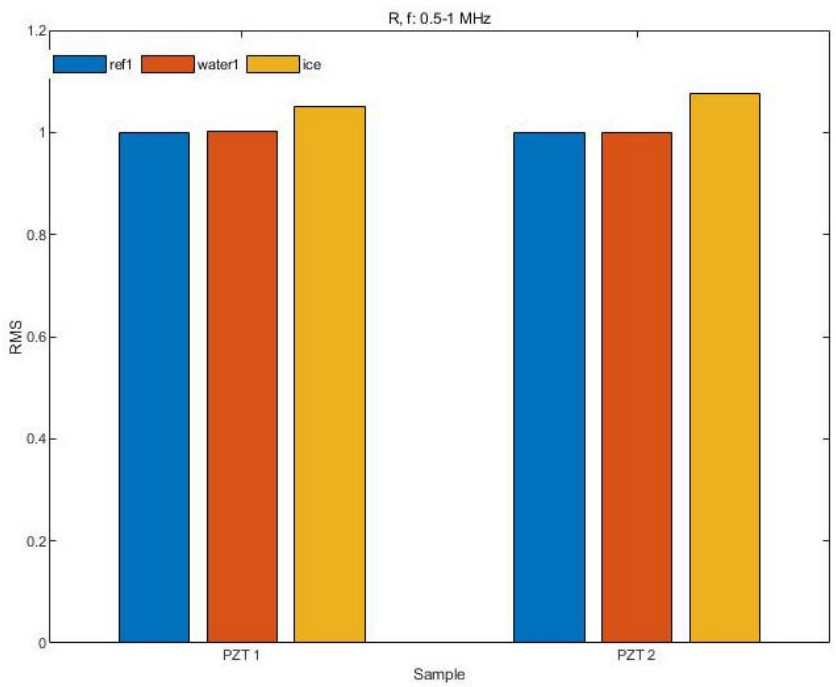

Fig. 8. RMS for resistance plots for quantification of changes in the three scenarios.

The GW based technique results are shown in Fig. 9. As can be seen the wave travel slightly slower with the introduction of the water. And the response is midway under the freezing conditions. This might be due to the temperature effect as well, and study in this respect is on-going. There is significant damping due to the water and the ice, which is in keeping with the observation made for the other plate as well as those reported in the literature [9].

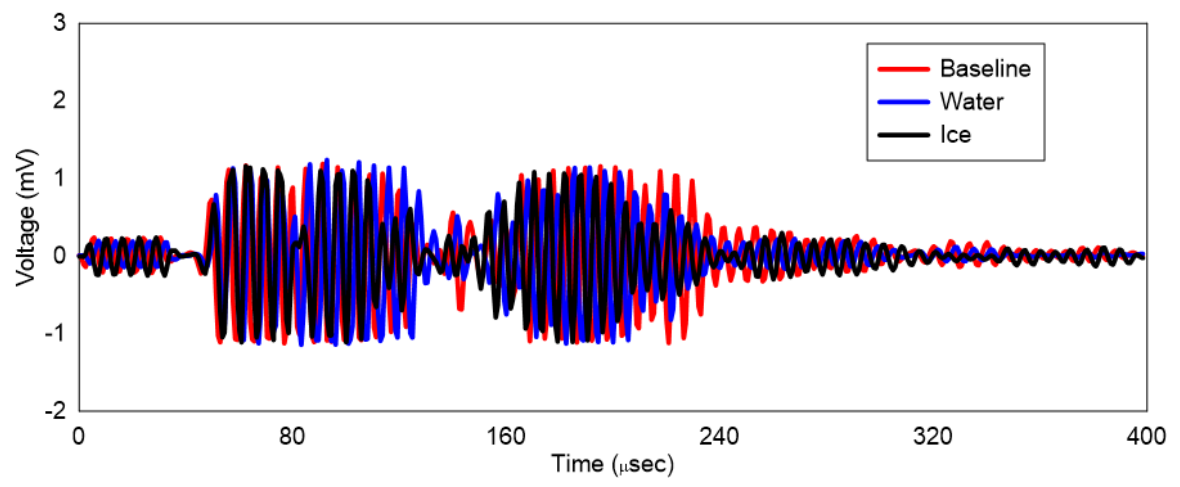

Fig. 9. GW propagation for dry sample, saturated and after icing for slotted plate. 


\section{Conclusion}

The paper aims at studying the deterioration of composite plates due to the formation of ice. 3 methods are used, unfortunately, due to the systematic errors of the measurement technique, the strain measurements yield unreliable results. The GW and EMI based technique yield reasonable results, and based on the basic processing, it seems that the icing and water ingress can both be detected by both the techniques. The comparative performance of the three techniques can be summarized as in Table 2. The use of temperature compensation is necessary and identified as the area of further work.

Table 2. Comparative performance of the three techniques

\begin{tabular}{|c|c|c|}
\hline Technique & Water & Ice \\
\hline EMI & $\checkmark$ & $\checkmark$ \\
\hline GW & $\checkmark$ & $\checkmark$ \\
\hline NA & $\times$ & $\times$ \\
\hline
\end{tabular}

In order to draw more conclusions additional experiments with Laser Doppler Vibrometer is necessary, which will allow more insight into the wave propagation and is identified as an area for future work. Also, development of algorithms for the quantification of the changes in the time of flight (similar to RMSD for EMI) is required for quantifying the changes.

\section{Acknowledgment}

The authors would like to acknowledge the National Science Center, Poland for their project grant no. 2015/17/N/ST8/01166, The authors are also grateful to TASK-CI for allowing the use of their computational resources.

\section{References}

1. S. Pant, PhD Dissertation," Carleton University Ottawa, Ontario, (2014).

2. D. Scida, M. Assarar, C. Poil`ane, and R. Ayad, Comp. Part B: Engg. 48, \{51-58\}, (2013).

3. V. Giurgiutiu and C. Rogers, SHM Current Status and Perspective, $\{18-20\}$, (1997).

4. V. Giurgiutiu, "Structural health monitoring: with piezoelectric wafer active sensors", Academic Press, (2007).

5. R. Soman, P. Malinowski, W Ostachowicz, Wind Energy, 19 (4), \{639-650\} (2016).

6. R. Soman, P. Malinowski, and W. Ostachowicz, in Proc. of the 7th EASN, (2017).

7. R. Soman, K. Majewska, M. Mieloszyk, P. Malinowski, and W. Ostachowicz, ejournal of NDT, (2016).

8. R. Soman, P. Malinowski, and W. Ostachowicz, In Health Monit. of Str. Bio. Sys. Vol. 10600, (2018).

9. K. Schubert and A. Herrmann, Comp. Struct, 94(12), (2012)

10. P. Malinowski, T. Wandowski, and W. Ostachowicz, Structural Health Monitoring, 14(4), pp.332-344. 